\title{
"Reverse J Shaped" Skin Flap and Cranial Bone Groove for Shunt Hardware: A New Technique for Patients with Fragile Skin
}

\author{
Murteza CAKIR \\ Ataturk University, Faculty of Medicine, Department of Neurosurgery, Erzurum, Turkey \\ Corresponding author: Murteza CAKIR murtezacakir28@gmail.com
}

\section{ABSTRACT}

Shunt systems are not perfect devices and can cause severe complications. Complications may originate from problems related to the valve, the patient, or the surgery and are more common in neonates, infants, especially preterm, by reason of the special characteristics of these patients. We, therefore, have successfully developed a useful and viable surgical technique in order to provide helpful aspects to the surgical issues mentioned above. This technique includes a "reverse J shaped" skin incision nonintersecting the shunt's hardware and distal catheter to reduce wound problems to a minimum by opening a "bone groove". We believe that the above technique described by us will both reduce issues such as scalp ulceration, wound dehiscence, and shunt exposure in premature and pediatric hydrocephalus cases with a friable scalp, and eliminate a cosmetic problem in hydrocephalic patients of all ages.

KEYWORDS: Bone groove, Hydrocephalus, Shunt operation, Surgical technique

\section{INTRODUCTION}

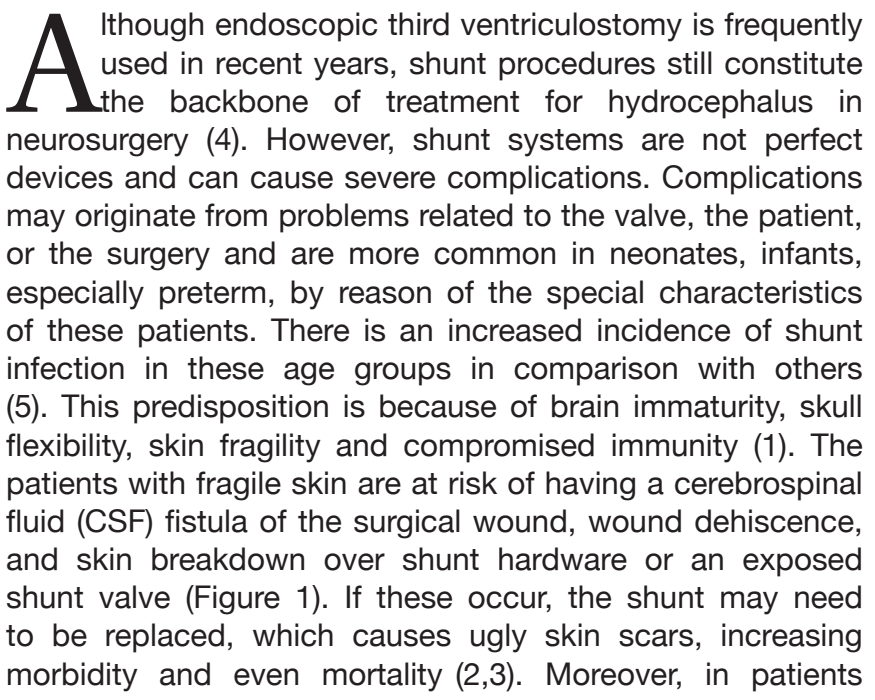

(adult or children) recovering without any wound problem, the shunt system raised from the skin also causes an unaesthetic appearance of the skull.

We, therefore, have successfully developed a useful and viable surgical technique in order to provide helpful aspects to surgical issues mentioned above.

\section{SURGICAL TECHNIQUE}

\section{Skin Flap Opening}

After induction of general anesthesia and surgical positioning, the point to which the proximal catheter is advanced is calculated by the computed tomography findings and a skin mark is made at a site corresponding to a proximal part of the lateral ventricle's occipital horn. With this point forming the center, two 5-cm lines are drawn, one in the direction of catheter's course, and the other perpendicular to the center (to form a crucifix). The intersection point of the perpendicular lines should be marked as the catheter's entry point. The two 
ends of the horizontal line are joined to form the upper part of a circle. Lastly, a $3-4 \mathrm{~cm}$ line, which is parallel to the distal catheter, is drawn from the left hand side of the horizontal line so that an incision line is formed in an "inverse J" shape. This pattern should constitute a mirror image, depending on the operation site being on the left- or right hand side of cranium (Figure 2). First, the skin flap is opened by tracking the "inverse J" shape. Then, the abdominal cavity is opened, and the distal catheter is sent through the tunnel from abdomen

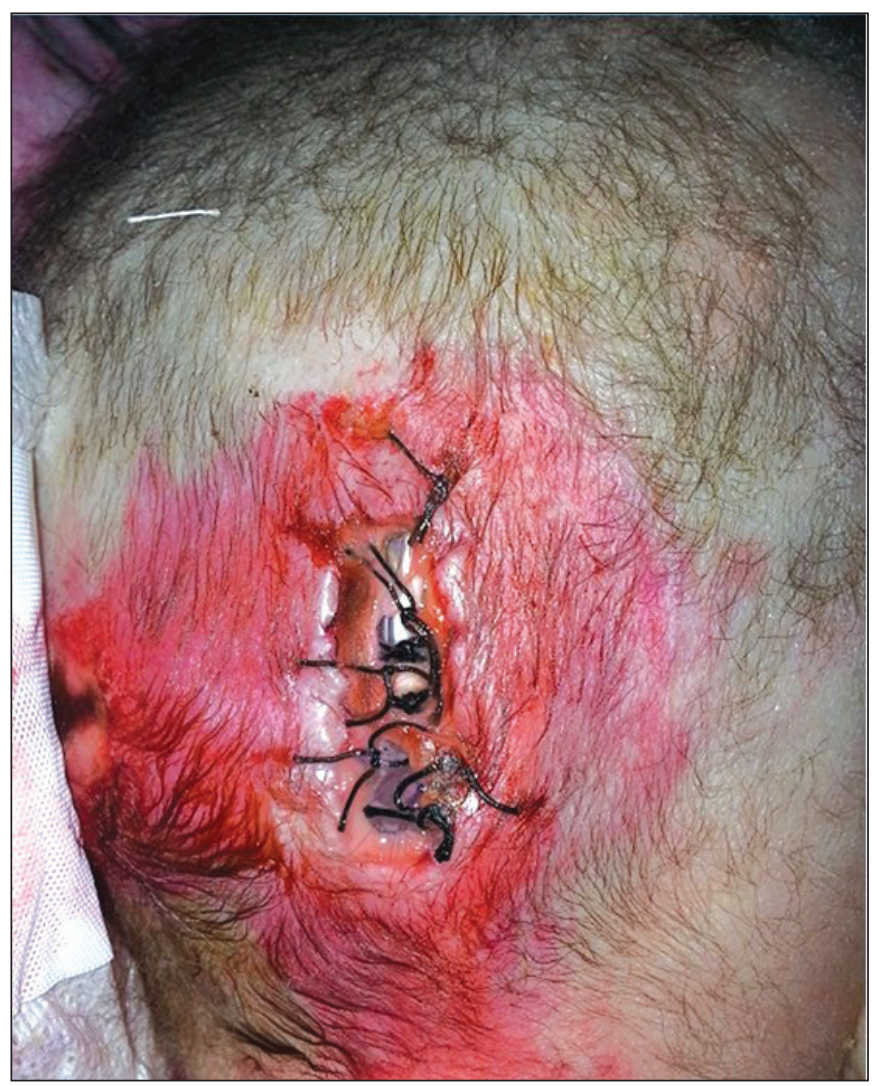

Figure 1: VP shunt wound dehiscence and exposed shunt.

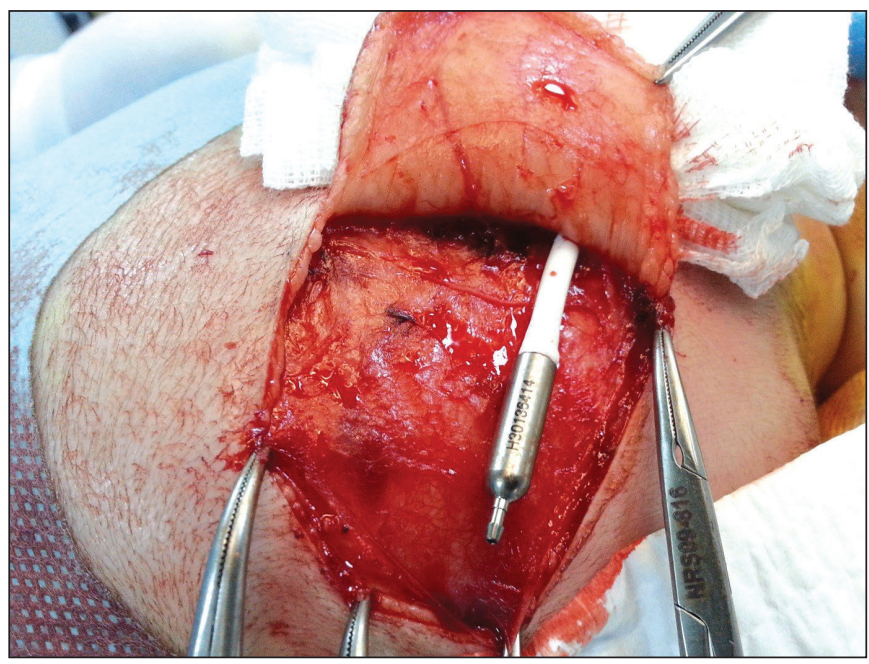

Figure 3: Placement of the distal catheter over the periosteum. to the cranial flap and passed beneath the flap and on the periosteum (Figure 3).

\section{Bone Groove Opening}

The periosteal flap is opened in a suitable manner for the skin flap. A small incision is made in the periosteum and the distal catheter is placed underneath the periosteum (Figure 4). A burr hole is opened from the projection of the ventricular catheter's entry point on the bone, which is marked on the skin. Depending on the shape, width, and length of the shunt hardware, a suitable bone groove is tailored by using a bone rongeur or a high-speed drill in the calvarium in the direction of the valve and/or reservoir system (Figure 5). Holes for suturing are opened to both edges of the sulcus with a high-speed drill. After placement and interconnection of the ventricular catheter with the valve, the catheter is drawn from its abdominal side to insert the hardware into the opened hole. The hardware is secured with sutures from two sites, each of which are passed through the hole in the bone and stabilized (Figure 6). With this sulcus opened by high-speed

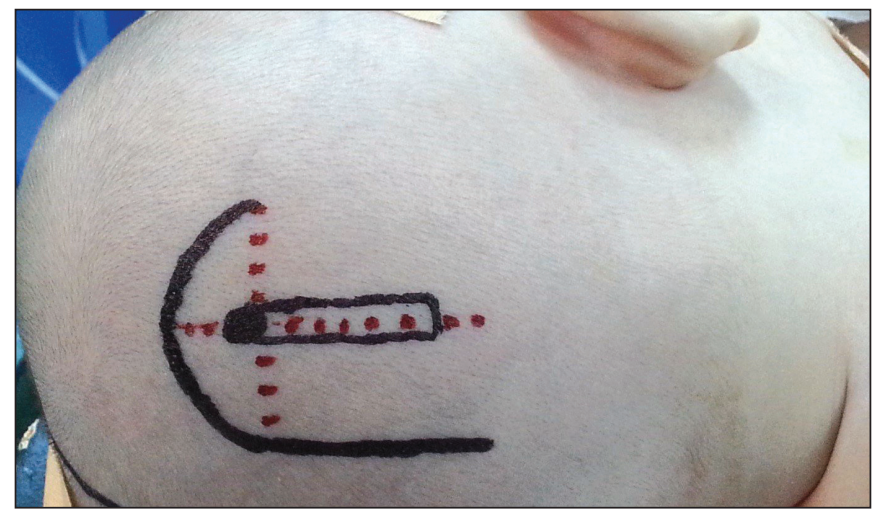

Figure 2: "Reverse J shaped" cranial skin flap.

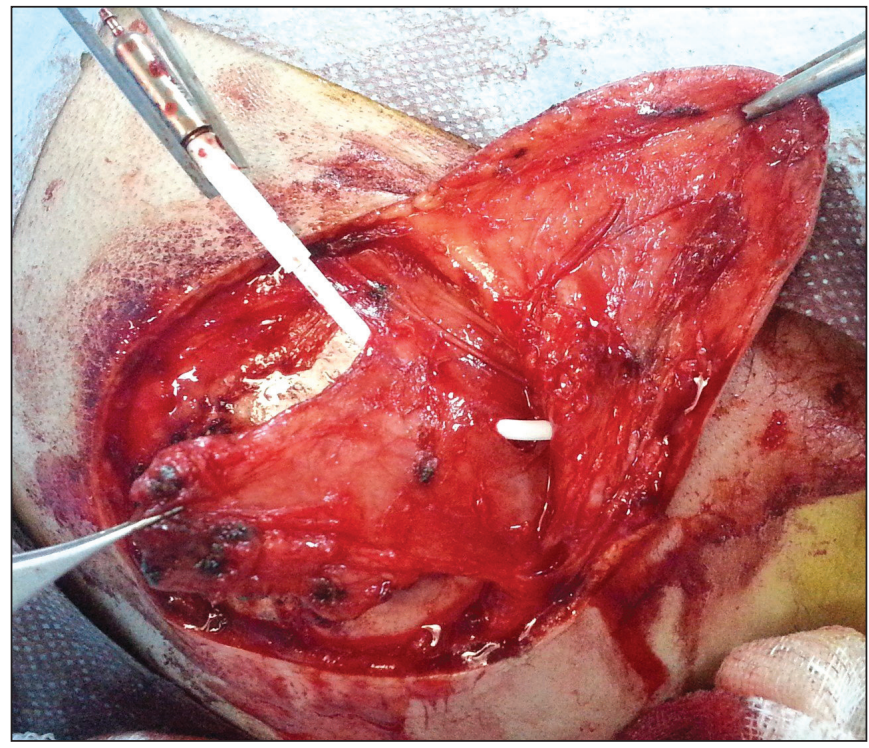

Figure 4: Placement of the distal catheter beneath the periosteum by an incision made in the periosteum. 
drill and/or rongeur, we obtain a zero- or low-profile shunt valve bed. We observed that good outcomes are achieved in terms of wound healing when suitable beds for both valve and reservoir are opened in shunts with monoblock valve and reservoir assembly, and when the reservoir is removed from the system and only a valve bed is opened in systems with separate reservoirs and valve. After advancing the distal end, the abdominal skin is closed. The periosteal flap covered on the hardware and sutured end-to-end, followed by closure of the skin flap (Figure 7 and 8). Embedding the valve into the cranium and closing the periosteum and scalp using a monolayer suture with an appropriate thickness improves the scalp's blood supply. By this way, the hardware becomes indiscernible by palpation and inspection. This prevents skin pressure and provides a good cosmetic outcome.

\section{- DISCUSSION}

One of the most important causes of shunt complications is shunt infection (6). Shunt infections often occur through bacterial contamination of cranial wounds that do not recover. Patients with fragile skin are particularly predisposed to wound dehiscence, exposed shunt and poor wound recovery $(1,2)$. Conventional shunt surgery negatively affects wound recovery of patients with poor skin. However, there are few alternative methods to conventional shunt surgery in the literature.

As emphasized in the subpericranial shunt valve replacement technique described by Gyang and colleagues, thinning of scalp tissue due to various reasons in hydrocephalic children may pose significant challenges after therapeutic shunt placement, such as failure of wound healing, development of decubitus ulcers, meningitis, and ventriculitis (2). To avoid these complications, we also place the shunt hardware into a pocket beneath the scalp. We have been applying this technique in our clinical practice for more than 20 years to prevent issues with blood supply to scalp that are a result of additional stress and local compression applied by the shunt hardware. Despite this, however, we may still encounter decubitus ulceration of scalp, surgical wound dehiscence and exposure of especially a shunt's valve region, and resulting

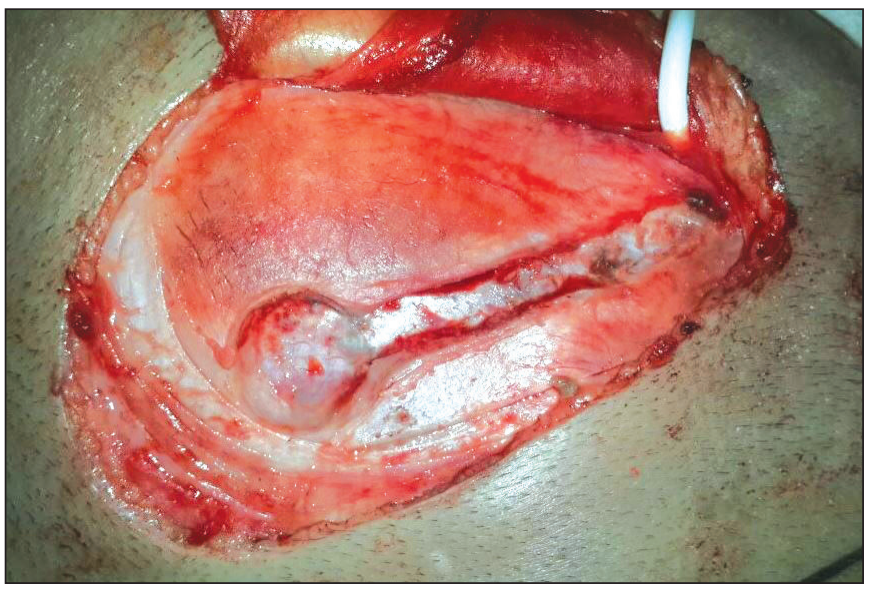

Figure 5: "Bone Groove" opened for the shunt hardware. infections after shunt applications with different models, both by us and others. Moreover, shunt hardware becoming visible from outside is a cosmetic problem in patients without wound problems. Hence, to prevent these serious medical and cosmetic problems, we have begun to apply a different surgical technique for 5 years.

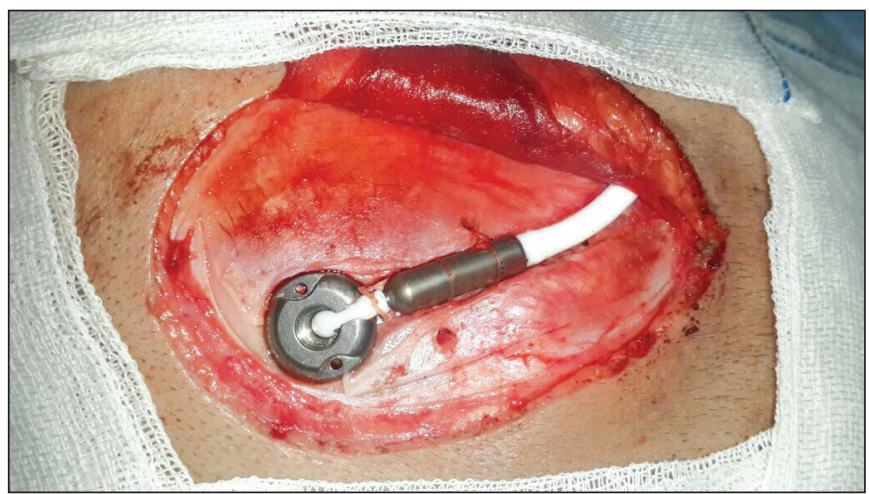

Figure 6: Placement of the shunt hardware into the bone groove and securing it to bone from both sides.

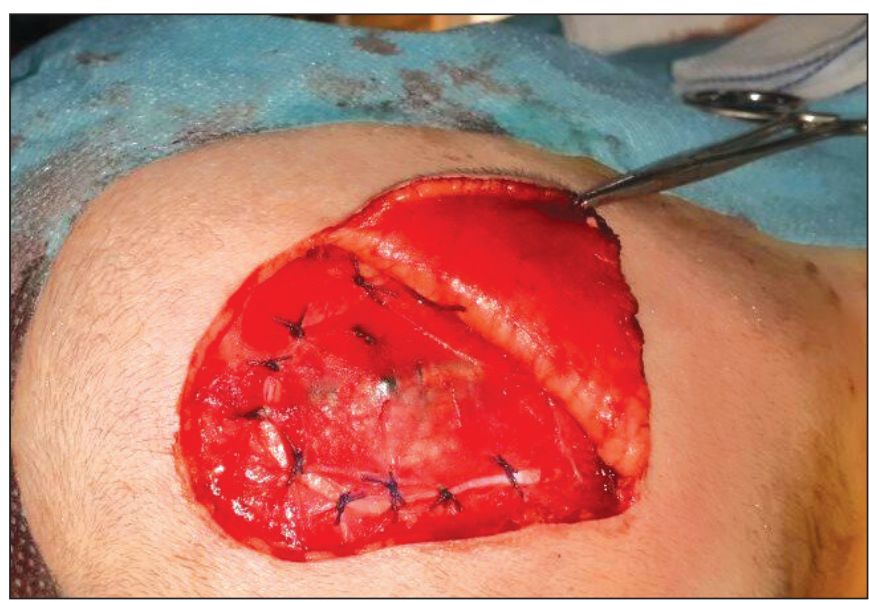

Figure 7: Covering periosteum on the shunt hardware after placing it into the bone groove.

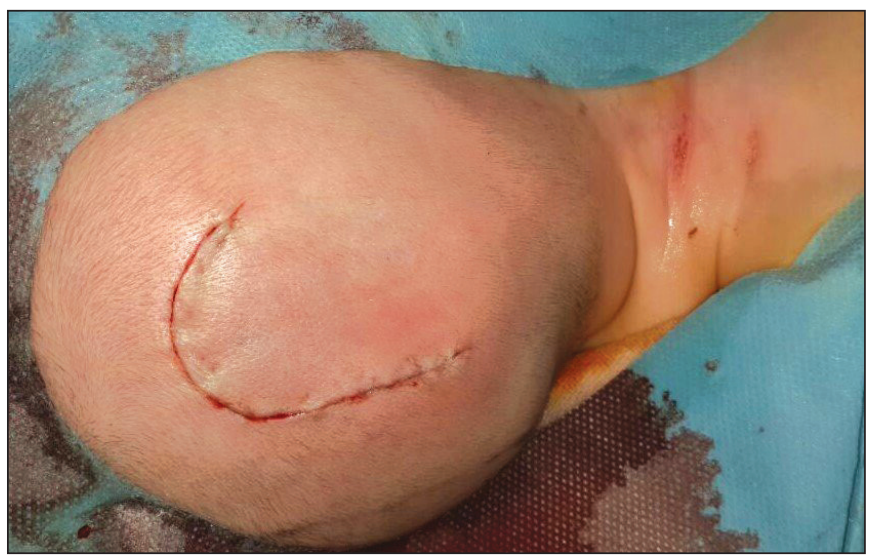

Figure 8: The closed skin flap. 
In the cranial incision of conventional shunt surgery, both the hardware and the distal catheter may intersect with the incision line and this may lead to poor wound healing, wound dehiscence or exposed shunt. However, our "reverse J shaped skin flap" technique has no negative effects on wound healing since no pieces of the shunt equipment coincide beneath the incision line. This technique enables wound healing with minimal scar tissue.

In the classical hardware replacement technique, both reservoir and hardware stay on the level of bone, causing tension and pressure on both the area of incision and on intact skin. So, this situation may lead to cranial shunt wound dehiscence, ventriculoperitoneal shunt-induced scalp necrosis, ulceration and exposed shunt. In our "cranial bone groove for shunt hardware" technique, the shunt hardware is placed into a custom-tailored bone groove, so it stays under the level of bone, causing relatively less tension or pressure on the overlying skin texture. Thanks to this technique, both wound dehiscence and exposed shunt related to skin necrosis is prevented, and healing of the primary wound enabled without issues. So this will both minimalize the cost raise morbidity and mortality caused by revision surgeries, through reducing shunt infection incidence related to exposed shunt and wound dehiscence and prevent the occurrence of unaesthetic appearances related to ugly skin scar tissue.

\section{- CONCLUSION}

We believe that this technique may reduce the incidence of issues such as scalp ulceration, wound dehiscence, and shunt exposure especially in premature cases and macrocephalic hydrocephalus cases with a friable scalp and may eliminate cosmetic problems in most of the pediatric hydrocephalus cases as well.

\section{- REFERENCES}

1. Ammar A, Nasser $M$ : A long-term complication of burying a shunt valve in the skull. Neurosurg Rev 18:65-67, 1995

2. Bot GM, Ismail NJ, Usman B, Shilong DJ, Obande JO, Aliu SO, Hassan I, Shehu BB: Subpericranial shunt valve placement: A technique in patients with friable skin. Childs Nerv Syst 30(8): 1431-1433, 2014

3. Gonzalez DO, Mahida JB, Asti L, Ambeba EJ, Kenney B, Governale L, Deans KJ, Minneci PC: Predictors of ventriculoperitoneal shunt failure in children undergoing initial placement or revision. Pediatr Neurosurg 52(1):6-12, 2017

4. Iglesias S, Ros B, Martín Á, Carrasco A, Segura M, Delgado A, Rius F, Arráez MÁ: Surgical outcome of the shunt: 15-year experience in a single institution. Childs Nerv Syst 32(12): 2377-2385, 2016

5. Raffa G, Marseglia L, Gitto E, Germanò A: Antibioticimpregnated catheters reduce ventriculoperitoneal shunt infection rate in high-risk newborns and infants. Childs Nerv Syst 31(7):1129-1138, 2015

6. Simon TD, Butler J, Whitlock KB, Browd SR, Holubkov R, Kestle JR, Kulkarni AV, Langley M, Limbrick DD Jr, MayerHamblett N, Tamber M, Wellons JC 3rd, Whitehead WE, Riva-Cambrin J: Risk factors for first cerebrospinal fluid shunt infection: Findings from a multi-center prospective cohort study. J Pediatr 164(6):1462-1468, 2014 